\title{
BETTI NUMBERS OF FIXED POINT SETS AND MULTIPLICITIES OF INDECOMPOSABLE SUMMANDS
}

\author{
SEMRA ÖZTÜRK KAPTANOĞLU
}

(Received 5 September 2001; revised 4 February 2002)

Communicated by S. Gradde

\begin{abstract}
Let $G$ be a finite group of even order, $k$ be a field of characteristic 2, and $M$ be a finitely generated $k G$-module. If $M$ is realized by a compact $G$-Moore space $X$, then the Betti numbers of the fixed point set $X^{C_{n}}$ and the multiplicities of indecomposable summands of $M$ considered as a $k C_{n}$-module are related via a localization theorem in equivariant cohomology, where $C_{n}$ is a cyclic subgroup of $G$ of order $n$. Explicit formulas are given for $n=2$ and $n=4$.
\end{abstract}

2000 Mathematics subject classification: primary 55M35; secondary $20 \mathrm{C} 05$.

Keywords and phrases: Betti number, fixed point set, Moore space, realizable module, multiplicity, indecomposable summand, localization, equivariant cohomology.

\section{Introduction}

Throughout the paper $G$ denotes a finite group of order divisible by a prime $p, A$ a subgroup of $G, k$ a field of characteristic $p, J$ the Jacobson radical of the group algebra $k G, M$ a finitely generated $k G$-module, $X$ a $G$-space, and $X^{A}$ the fixed point set of $A$ in $X$. Topological spaces with a $G$-action give rise to $G$-modules; for example, the cohomology group $H^{i}(X ; k)$ with $k$-coefficients is a finitely generated $k G$-module for $i \geq 0$ provided that $X$ is a compact $G$-space. Equivariant cohomology $H_{G}^{*}(X ; k)$ of $X$ is defined as the cohomology $H^{*}\left(X_{G} ; k\right)$ of the Borel construction $X_{G}=(X \times E G) / G$ of $X$. When $X$ is a point, we simply write $H_{G}^{*}$ for $H_{G}^{*}(X ; k)$ which is the same as $H^{*}(G ; k)$. The constant map from $X$ to a one-point space induces an $H_{G}^{*}$-module structure on $H_{G}^{*}(X ; k)$. When $G$ is an elementary abelian $p$-group and $X$ is finitedimensional, the inclusion map $j:\left(X^{G}, x_{0}\right) \hookrightarrow\left(X, x_{0}\right)$ induces an isomorphism in the localized equivariant cohomology of $H_{G}^{*}$-modules ([Qu]). A simply connected

(C) 2003 Australian Mathematical Society $1446-7887 / 03 \$ A 2.00+0.00$ 
$G$-space $X$ is called a $G$-Moore space if $H^{i}\left(X, x_{0} ; k\right)=0$ for all $i$ except for some fixed $n \geq 2$. A $k G$-module $M$ is called realizable (in dimension $n$ ) if there exists a $G$-Moore space $X$ whose cohomology in dimension $n$ is $M$ for some $n \geq 2$.

Suppose that $M$ is a $k G$-module realized by $X$ in dimension $n$. Then $M \downarrow_{k A}$, $M$ considered as a $k A$-module, is also realized by $X$, and $H^{*}(A ; M)$ is isomorphic to the equivariant cohomology ring $H_{A}^{*+n}\left(X, x_{0} ; k\right)$. Combining this with the above isomorphism obtained by localization, of course for a 'nice' $A$ or a 'nice' $A$-action (for example $A$ acting semi-freely on $X$, that is, the isotropy subgroups being either $A$ or $\{1\})$, we observe that the multiplicities of the indecomposable modules appearing in the decomposition of $M \downarrow_{k A}$ have a geometric interpretation in terms of the total Betti number $\beta$ of the fixed point set $X^{A}$.

THEOREM. Let $G$ be a finite group of order divisible by 2 , and $C$ be a cyclic subgroup of $G$. Suppose that $M$ is realized in dimension $n$ by a compact space $X$. Then the following can be stated for the total Betti number $\beta$ and the Euler characteristic $\chi$ of the fixed point set $X^{C}$ of $C$ :

(a) If $C \cong \mathbb{Z}_{2}$, then $\beta\left(X^{C}\right)=\eta_{1}+1$, where $M \downarrow_{k C} \cong(k)^{\eta_{1}} \oplus(k C)^{\eta_{2}}$.

(b) If $C \cong \mathbb{Z}_{4}$ and $C$ acts semi-freely on $X$, then

(i) $\beta^{\text {odd }}\left(X^{c}\right)$ is $\eta_{1}$ or $\eta_{3}$ if $n$ is odd or if $n$ is even, respectively, and $\beta\left(X^{c}\right)=$ $\eta_{1}+\eta_{3}+1$

(ii) $\chi\left(X^{C}\right)=(-1)^{n}\left(\eta_{1}-\eta_{3}\right)+1$,

where $M \downarrow_{k C} \cong(k)^{\eta_{1}} \oplus\left(J^{2}\right)^{\eta_{2}} \oplus(J)^{\eta_{3}} \oplus(k C)^{\eta_{4}}$.

The restriction on the order of the cyclic subgroup $C$ to be 2 or 4 in the theorem is due to the fact that for large orders that are powers of a prime $p \geq 2$, one could still obtain an isomorphism $H_{C}^{*}\left(X^{C}, x_{0} ; k\right)[1 / t] \cong H^{*}\left(C ; M \downarrow_{k C}\right)[1 / t]$. However, interpreting the right hand side of the isomorphism to obtain a similar formula is not possible without such restrictions.

A corollary of the theorem is given in the discussion section.

\section{Proof of Theorem}

DEFINITION. Let $S$ be a multiplicative subset of the polynomial part of $H_{G}^{*}$ containing $1 \in H_{G}^{*}$, and $G_{x}$ be the isotropy subgroup consisting of all $g \in G$ with $g x=x$. Define $X^{S}=\left\{x \in X: \operatorname{ker}\left\{\right.\right.$ res $\left.\left.: H_{G}^{*} \rightarrow H_{G_{x}}^{*}\right\} \cap S=\emptyset\right\}$ following [Hs].

In some cases $X^{S}$ turns out to be the same as the fixed point set $X^{A}$ for some $A \leq G$; see $[\mathrm{DW}]$. 
Proposition 1. Let $G$ be a compact Lie group, $X$ be a compact $G$-space, and $Y \subseteq X$ be a $G$-invariant subspace. Let $S \subset H_{G}^{*}$ be a multiplicative system. Then the localized homomorphism

$$
\rho^{-1}=S^{-1} i^{*}: S^{-1} H_{G}^{*}(X, Y) \rightarrow S^{-1} H_{G}^{*}\left(X^{S}, Y^{S}\right)
$$

is an isomorphism, where $i^{*}$ is the induced map in G-equivariant cohomology by the inclusion map $i:\left(X^{S}, Y^{S}\right) \hookrightarrow(X, Y)$.

PROOF. Recall that localization is an exact functor, and $\rho=S^{-1} i_{G}^{*}: S^{-1} H_{G}^{*}(X) \rightarrow$ $S^{-1} H_{G}^{*}\left(X^{S}\right)$ is an isomorphism, where $i_{G}^{*}$ is the map induced by the inclusion $i$ : $X^{S} \hookrightarrow X$ in $G$-equivariant cohomology. Apply [Hs, Theorem III.1] to the long exact sequence of a pair in cohomology. The result then follows by the Five-Lemma.

PROPOSITION 2. Let $M$ be a $k G$-module realized by $X$ in dimension $n$. Then $H_{G}^{*+n}\left(X, x_{0} ; k\right) \cong H^{*}(G ; M)$.

Proof. Consider the Serre spectral sequence for the fibration $\left(X, x_{0}\right)_{G}=\left(\left(X, x_{0}\right) \times\right.$ $E G) / G \rightarrow E G / G=B G$ with fiber $\left(X, x_{0}\right)$. Here $E G$ is a contractible space on which $G$ acts (fixed-point) freely. The spectral sequence has $E_{2}^{p, q}$-term equal to $H^{p}\left(G ; H^{q}\left(X, x_{0} ; k\right)\right)$. For $q \neq n$, we have $H^{q}\left(X, x_{0} ; k\right)=0$; then $E_{2}^{p . q}=0$ for $q \neq n$. Hence the sequence contains only one line and collapses. It follows that $E_{2}^{p, n}=$ $H^{p}\left(G ; H^{n}\left(X, x_{0} ; k\right)\right) \cong H^{p}(G ; M)$. Therefore $H_{G}^{*+n}\left(X, x_{0}\right):=H^{*+n}\left(\left(X, x_{0}\right)_{G} ; k\right) \cong$ $H^{*}(G ; M)$.

ProOF OF THEOREM. Without loss of generality we may assume that $X^{G}$ is nonempty; so let $x_{0}$ be in $X^{G} \subseteq X^{K}$ for $K \leq G$. Also $X$ is a $K$-Moore space with $H^{*}\left(X ; x_{0}\right) \cong M \downarrow_{k K}$ for $K \leq G$. Hence $H_{K}^{*+n}\left(X, x_{0}\right) \cong H^{*}\left(K ; M \downarrow_{k K}\right)$ by Proposition 2.

(a) Let $H_{C}^{*}=H^{*}(C ; k)=k[t]$. By Proposition 1 , localization with respect to $S=\left\{t^{i}: i \geq 0\right\}$ gives $H_{C}^{*}\left(X, x_{0}\right)[1 / t] \cong H_{C}^{*}\left(X^{C}, x_{0}\right)[1 / t]$. Since res ${ }_{c, 1\}}(t)=$ 0 , we have $k[1 / t]=0$. Hence $\eta_{2}$ disappears after localization and we obtain $\operatorname{dim}_{k} H^{*}\left(X^{c}, x_{0} ; k\right)=\beta\left(X^{c}\right)-1=\eta_{1}$, that is, $\beta\left(X^{C}\right)=\eta_{1}+1$.

(b) It is sufficient to prove only (i) since $X\left(X^{C}\right)=\beta^{\text {even }}\left(X^{C}\right)-\beta^{\text {odd }}\left(X^{C}\right)$. Let $C_{2} \leq$ $C$ and $C_{2} \cong \mathbb{Z}_{2}$; let also $H_{C}^{*}=k\left[\tau^{\prime}\right] \otimes \wedge\left(v^{\prime}\right)$ and $H_{C_{2}}^{*}=k[t]$. Thus res ${ }_{C, c_{2}}\left(\tau^{\prime}\right)=t^{2}$. We have $H^{*}\left(C ; M \downarrow_{k C}\right) \cong\left(H_{C}^{*}\right)^{\eta_{1}} \oplus\left(H_{C_{2}}^{*}\right)^{\eta_{2}} \oplus\left(H^{*}(C ; J)\right)^{\eta_{3}} \oplus(k)^{\eta_{4}}$ since $J^{2} \cong k\left[C / C_{2}\right] \cong$ $k \uparrow_{k C_{2}}^{k C}$ and Shapiro's Lemma implies $H_{C_{2}}^{*} \cong H^{*}\left(C ; J^{2}\right)$. Applying Proposition 1 with the multiplicative set $S=\left\{\left(\tau^{\prime}\right)^{i}: i \geq 0\right\}$ gives $H_{C}^{*}\left(X^{C_{2}}, x_{0}\right)\left[1 / \tau^{\prime}\right] \cong H_{C}^{*}\left(X, x_{0}\right)\left[1 / \tau^{\prime}\right]$. The term with $\eta_{4}$ disappears after localization as in part (a). Hence

$$
H_{C}^{*}\left(X^{C_{2}}, x_{0}\right)\left[\frac{1}{\tau^{\prime}}\right] \cong\left(H_{C}^{*}\left[\frac{1}{\tau^{\prime}}\right]\right)^{\eta_{1}} \oplus\left(H_{C_{2}}^{*}\left[\frac{1}{t^{2}}\right]\right)^{\eta_{2}} \oplus\left(H^{*}(C ; J)\left[\frac{1}{\tau^{\prime}}\right]\right)^{\eta_{3}}
$$


The hypothesis that $C$ acts semi-freely on $X$ implies $X^{C}=X^{C_{2}}$. Write $\hat{H}_{C}^{*}=H_{C}^{*}\left[1 / \tau^{\prime}\right]$ and $\hat{H}_{C_{2}}^{*}[1 / t]$. Then

$$
\left(\hat{H}_{C}^{*-n}\right)^{\eta_{1}} \oplus\left(\hat{H}_{C_{2}}^{*-n}\right)^{\eta_{2}} \oplus\left(H^{*-n}(C ; J)\left[\frac{1}{\tau^{\prime}}\right]\right)^{\eta_{3}} \cong H^{*}\left(X^{C}, x_{0}\right) \otimes \hat{H}_{C}^{*}
$$

Since $H^{i}(C ; J) \cong H^{i-1}(C ; k)=H_{C}^{i-1}$ for $i \geq 2$ and $H_{C}^{\text {ddd }}=v^{\prime} H_{C}^{\text {even }}$, we get $H^{i}(C ; J) \cdot v^{\prime}=0$ for $i$ even. Also $H_{C_{2}}^{*} \cdot v^{\prime}=H_{C_{2}}^{*} \cdot \operatorname{res}_{C_{1} C_{2}}\left(v^{\prime}\right)=H_{C_{2}}^{*} \cdot 0=0$. Then $(*)$ becomes

$$
\left(\hat{H}_{C}^{l-n} \cdot v^{\prime}\right)^{\eta_{1}} \oplus\left(\hat{H}_{C}^{l-n-1} \cdot v^{\prime}\right)^{\eta_{3}} \cong \sum_{i \geq 0, i \text { even }}^{l} H^{l-i}\left(X^{C}, x_{0}\right) \otimes \hat{H}_{C}^{i} \cdot v^{\prime} .
$$

In particular,

$$
\sum_{j \geq 0, j \text { even }}^{l} H^{l-j}\left(X^{c}, x_{0}\right) \otimes \hat{H}_{C}^{j} \cdot v^{\prime} \cong \begin{cases}(k)^{\eta_{3}}, & \text { if } l-n \text { is odd } \\ (k)^{\eta_{1}}, & \text { if } l-n \text { is even. }\end{cases}
$$

Choose an integer $l>\operatorname{Hom} \operatorname{dim}\left(X^{c}\right)$. For $l$ even and $l$ odd, we respectively obtain that

$$
\beta^{\text {even }}\left(X^{c}\right)= \begin{cases}\eta_{3}+1, & \text { if } n \text { is odd } \\ \eta_{1}+1, & \text { if } n \text { is even }\end{cases}
$$

and

$$
\beta^{\text {odd }}\left(X^{c}\right)= \begin{cases}\eta_{1}, & \text { if } n \text { is odd } \\ \eta_{3}, & \text { if } n \text { is even. }\end{cases}
$$

This completes the proof of the theorem.

\section{Discussion}

The theorem of the paper is more meaningful when put in the context of the realization problem referred to in the literature as Steenrod's Problem, and/or in the classification problem of some category of $k G$-modules when $G$ contains cyclic subgroups of order 2 and/or 4 . (See the corollary below.) When $G$ is a cyclic $p$ group of order $p^{n}$, all indecomposable $k G$-modules (up to isomorphism) are given by the powers of the Jacobson radical, namely, the ideals $J^{p^{n}-i}$ of $k$-dimension $i$ for $i=1, \ldots, p^{n}$. However, when $G$ contains $\mathbb{Z}_{p} \times \mathbb{Z}_{p}$ there are infinitely many indecomposable $k G$-modules ([Hi]). Due to the lack of a classification for $k G$ modules when $G \supseteq \mathbb{Z}_{p} \times \mathbb{Z}_{p}$ except for $G=\mathbb{Z}_{2} \times \mathbb{Z}_{2}$, considering the restrictions $M \downarrow_{k A}$ for various subgroups $A$ in $G$ to obtain information on $M$ is a fundamental technique 
$\mathrm{n}$ modular representation theory. For example, the complexity of a $k G$-module, n particular, the cohomology $H^{*}(G ; k)$ of the trivial $k G$-module $k$ is 'detected' on maximal elementary abelian subgroups of $G$ by theorems due to Quillen [Qu], Thouinard [Ch], and Alperin-Evens [AlEv]. See [Ka] for another detection theorem when $G=\mathbb{Z}_{2} \times \mathbb{Z}_{4}$. Furthermore, it is possible to obtain information on a $k E$-module $M$ by considering $M \downarrow_{k(1+x)}$ for $x \in J \backslash J^{2}$ of $k E$, where $E$ is an elementary abelian D-group [Ca]. See also [W].

Some partial results on Steenrod's Problem are as follows. All $k \mathbb{Z}_{p^{m}}$-modules are ealizable (see [Ar]) and all realizable $k \mathbb{Z}_{2} \times \mathbb{Z}_{2}$-modules are described in [BeHa]. When $\mathbb{Z}_{2} \times \mathbb{Z}_{2}$ is a normal Sylow subgroup of a finite group $G$, a $k G$-module $M$ is ealizable if and only if $M \downarrow_{k \mathbb{Z}_{2} \times \mathbb{Z}_{2}}$ is realizable ([Cn]). When $G$ contains $\mathbb{Z}_{p} \times \mathbb{Z}_{p}$, there are $k G$-modules that are not realizable (see [Vo, Cs, As1, As2, BeHa]). Compare our :heorem with [As3, Theorem 2.2], which states that the total Betti number $\beta\left(X^{A}\right)$ of a 'nice' Moore space $X$ realizing a $k E$-module $M$ is equal to the $\operatorname{rank}\left(\mathscr{F}_{A}\right)$, where $\mathscr{F}_{A}$ is the characteristic sheaf of $X$ and $A$ is a subgroup of the elementary abelian $p$-group $E$.

The simplest group for which one can attack the classification problem or the realization problem for $k G$-modules is $G=\mathbb{Z}_{2} \times \mathbb{Z}_{4}$ due to the fact that it contains $\mathbb{Z}_{2} \times \mathbb{Z}_{2}$ as its unique maximal elementary abelian subgroup and that the classification of $k \mathbb{Z}_{2} \times \mathbb{Z}_{2}$-modules is known. As mentioned above, a 'detection' theorem supporting the first expectation is given in [Ka]. For the latter, we can only give a necessary condition for a $k \mathbb{Z}_{2} \times \mathbb{Z}_{4}$-module $M$ to be realizable by combining [Cs, Proposition II] and [Se, Proposition 1]: Let $M$ be a $k \mathbb{Z}_{2} \times \mathbb{Z}_{4}$-module. If $M \downarrow_{k \mathbb{Z}_{2} \times \mathbb{Z}_{2}}$ is realizable by $X$, then the rank variety $V_{\mathbb{Z}_{2} \times \mathbb{Z}_{2}}^{r}\left(M \downarrow_{k \mathbb{Z}_{2} \times \mathbb{Z}_{2}}\right.$ ) (see [Ca]) is a union of $\mathbb{F}_{2}$-rational lines in $k^{2}$. Therefore for a realizable $k \mathbb{Z}_{2} \times \mathbb{Z}_{4}$-module $M$, we obtain that $M \downarrow_{k S}$ is free for every shifted cyclic subgroup $S$ of $k \mathbb{Z}_{2} \times \mathbb{Z}_{4}$ except possibly for cyclic subgroups of $\mathbb{Z}_{2} \times \mathbb{Z}_{4}$. This can be used to construct non-realizable modules. Consider the induced $k \mathbb{Z}_{2} \times \mathbb{Z}_{4}$-module $M_{\alpha}=k \otimes_{k\left\langle u_{\alpha}\right\rangle} k \mathbb{Z}_{2} \times \mathbb{Z}_{4}$ for $\alpha \in k^{2}$. It can be seen easily by Mackey's formula that $V_{\mathbb{Z}_{2} \times \mathbb{Z}_{2}}^{r}\left(M_{\alpha} \downarrow_{k \mathbb{Z}_{2} \times \mathbb{Z}_{2}}\right)=k\{\alpha\}$ for $\alpha \in k^{2}$. Therefore, $M_{\alpha}$ is not realizable if $\alpha$ is not an $\mathbb{F}_{2}$-rational point.

The Theorem of this paper and the necessary condition mentioned above gives the following.

COROLlary. Let $G=\left\langle e, f: e^{2}=f^{4}=e f e f^{3}=1\right\rangle \supset E=\left\langle e, f^{2}\right\rangle$. If $M$ is a non-free indecomposable $k G$-module realized by $X$, then $M$ is a periodic $k G$ module, and $M \downarrow_{k\left(1+\alpha_{1}(e-1)+\alpha_{2}\left(f^{2}-1\right)\right)}$ is a free $k\left(1+\alpha_{1}(e-1)+\alpha_{2}\left(f^{2}-1\right)\right\rangle$-module for $\left(\alpha_{1}, \alpha_{2}\right) \in k^{2}$ except possibly for $\left(\alpha_{1}, \alpha_{2}\right) \in k\{(1,0)\} \cup k\{(0,1)\} \cup k\{(1,1)\}$. Moreover, if $M \downarrow_{k(g)}$ is a free $k\langle g\rangle$-module for $g \in\left\{e, f^{2}, e f^{2}\right\}$, then $X^{\langle g\rangle}$ is homotopic to a point.

Proof. The necessary condition given above for the realizability of a module $M$ implies that $V=V_{E}^{r}\left(M \downarrow_{k E}\right) \subseteq k\{(1,0)\} \cup k\{(0,1)\} \cup k\{(1,1)\}$. This forces $M$ to 
be periodic as it is indecomposable and non-free. In addition, since $k\left\langle 1+\alpha_{1}(e-\right.$ 1) $\left.+\alpha_{2}\left(f^{2}-1\right)\right\rangle$ for $\alpha \in\{(1,0)\} \cup k\{(0,1)\} \cup k\{(1,1)\}$ corresponds to $k\langle g\rangle$ for some $g \in\left\{e, f^{2}, e f^{2}\right\}$, it follows that $M \downarrow_{\langle g\rangle}$ is not free for at most one $g \in\left\{e, f^{2}, e f^{2}\right\}$. Suppose $M \downarrow_{\langle g\rangle}$ is a free $k\langle g\rangle$-module with $g \in\left\{e, f^{2}, e f^{2}\right\}$. Then it has no trivial summands, that is, $\eta_{1}=0$. Hence $\beta\left(X^{\langle g\rangle}\right)=1$ by the theorem, and this implies that $X^{\langle g\rangle}$ is homotopic to a point.

CONJECTURE. If $M$ is a finitely generated periodic $k \mathbb{Z}_{2} \times \mathbb{Z}_{4}$-module, then $M$ is realizable.

\section{Acknowledgement}

I am indebted to Professor A. Assadi for introducing this subject and sharing ideas with me.

\section{References}

[AlEv] J. L. Alperin and L. Evens, 'Representations, resolutions, and Quillen's dimension theorem', J. Pure Appl. Algebra 144 (1981), 1-9.

[Ar] J. E. Arnold, 'On Steenrod's problem for cyclic $p$-groups', Canad. J. Math. 29(1977), 421-428.

[Asl] A. H. Assadi, 'Varieties in finite transformation groups', Bull. Amer. Math. Soc. 19 (1998), $459-463$.

[As2] - Homotopy actions and cohomology of finite transformation groups, Lecture Notes in Math. 1217 (Springer, Berlin, 1986), pp. 26-57.

[As3] - 'Algebraic geometric invariants for homotopy actions', in: Prospects in topology (Princeton, 1994), Ann. of Math. Stud. 138 (Princeton Univ. Press, Princeton, 1995) pp. 13-27.

[BeHa] D. Benson and N. Habbager, 'Varieties for modules and a problem of Steenrod', J. Pure Appl. Algebra 44 (1987), 13-34.

[Ca] J. F. Carlson, 'The variety and the cohomology ring of a module', J. Algebra 85 (1983), 104-143.

[Ch] L. Chouinard, 'Projectivity and relative projectivity for group rings', J. Pure Appl. Algebra 7 (1976), 287-302.

[Cn] M. Chen, 'The Postnikov tower and the Steenrod problem', Proc. Amer. Math. Soc. 129 (2001), 1825-1831.

[Cs] G. Carlsson, 'A counterexample to a conjecture of Steenrod', Invent. Math. 64(1981), 171-174.

[DW] W. G. Dwyer and C. W. Wilkerson, 'Smith theory revisited', Ann. of Math. (2) 127 (1988), 191-198.

[Hi] D. G. Higman, 'Indecomposable representations at characteristic p', Duke Math. J. 21 (1954), 377-381.

[Hs] W. Y. Hsiang, Cohomology theory of topological transformation groups (Springer, Berlin, 1975). 
[Ka] S. Ö. Kaptanoğlu, 'A detection theorem for $k \mathbb{Z}_{2} \times \mathbb{Z}_{4}$-modules via shifted cyclic subgroups', (preprint).

[Qu] D. Quillen, 'The spectrum of an equivariant cohomology ring I, II', Ann. of Math. (2) 94 (1971), 549-602.

[Se] J. P. Serre, 'Sur la dimension cohomologique des groupes profinis', Topology 3 (1965), 413 420.

[Vo] P. Vogel, 'A solution to the Steenrod problem for $G$-Moore spaces', $K$-Theory 1 (1987), 325-335.

[W] W. W. Wheeler, 'The generic module theory', J. Algebra 183 (1996), 205-228.

\section{Mathematics Department}

Middle East Technical University

Ankara 06531

Turkey

e-mail: semra@arf.math.metu.edu.tr 
